\title{
Forces for the particles with a zero energy in Kerr metric
}

\author{
A. A. Grib ${ }^{1}$, V. D. Vertogradov ${ }^{\bowtie 1,2}$, L. A. Shleiger ${ }^{1}$ \\ ${ }^{1}$ Herzen State Pedagogical University of Russia, 48 Moika Emb., Saint Petersburg 191186, Russia \\ ${ }^{2}$ The SAO RAS, Pulkovskoe shosse 65, Saint Petersburg 196140, Russia
}

\begin{abstract}
Authors
Andrey A. Grib, ORCID: 0000-0002-6389-991X, e-mail: andrei_grib@mail.ru

Vitalii D. Vertogradov, ORCID: 0000-0002-5096-7696, e-mail: vdvertogradov@gmail.com

Leonid A. Shleiger, e-mail: 1slejger@gmail.com

For citation: Grib, A. A, Vertogradov, V. D., Shleiger, L. A. (2021) Forces for the particles with a zero energy in Kerr metric. Physics of Complex Systems, 2 (4), 180-184. https://www.doi.org/10.33910/2687-153X-2021-2-4-180-184

Received 21 September 2021; reviewed 28 September 2021; accepted 28 September 2021.

Funding: The work was performed within the SAO RAS state assignment in the part "Conducting Fundamental Science Research".

Copyright: ( $)$ A. A. Grib, V. D. Vertogradov, L. A. Shleiger (2021). Published by Herzen State Pedagogical University of Russia. Open access under CC BY-NC License 4.0.
\end{abstract}

\begin{abstract}
The particles with negative energy might exist in the ergosphere of a rotating black hole due to collision or decay. We also know that there might be particles with zero energy in this region. In this paper we find out how the geodesic equations depend upon the energy and consider forces which act on the particles with zero energy. Also, we investigate the question how these forces for particles with zero energy differ from the ones in the case of usual positive energy $E$. We find out that the forces in the usual case are less than in the case of zero energy.
\end{abstract}

Keywords: Black hole, Kerr spacetime, ergosphere, zero energy, forces.

\section{Introduction}

In 1969, Penrose theorized an effect (Penrose 1969) which was called the Penrose process. According to this effect the particles with negative energy can exist in the ergosphere of a rotating black hole due to decay or collision. The thorough analysis of geodesics for such particles has been done in (Grib et al. 2014). Later, it was understood that there might be particles with zero energy (Grib, Pavlov 2017). This effect is possible due to the fact that the Killing vector $\frac{d}{d t}$ is spacelike in the ergosphere and the parameter $\mathrm{E}$, which is the energy with regard to infinity, might be either negative or equal to zero (Vertogradov 2015). If we consider the second order geodesic equation, then we obtain Newton's second law of mechanics and it is very interesting how negative or zero energy changes the forces which act on the particle. In this paper we will consider only radial movement in the equatorial plane $\theta=\pi / 2$ and forces which act on the particle with zero energy.

The paper is organized as follows. In Section 2 we will discuss some properties of the Kerr metric and obtain the geodesic equation in this spacetime. Section 3 represents the discussion of the forces which act on the particle with zero energy and their difference to usual case of positive energy. Section 4 is the conclusion.

\section{The Kerr metric. General notes}

A metric of a neutral rotating black hole is a well-known solution of the Einstein equation obtained by Kerr (Teukolsky 2015). The Kerr metric in Boyer-Lindquist coordinates has the following form (Chandrasekhar 1983; Poisson 2004; Vladimirov 2009): 


$$
\begin{gathered}
d s^{2}=-\left(1-\frac{2 M r}{\rho^{2}}\right) d t^{2}-\frac{4 M r a \sin ^{2} \theta}{\rho^{2}} d t d \varphi+\frac{\rho^{2}}{\Delta} d r^{2}+\rho^{2} d \theta^{2} \\
+\left(r^{2}+a^{2}+\frac{2 M r a^{2} \sin ^{2} \theta}{\rho^{2}}\right) \sin ^{2} \theta d \varphi^{2}
\end{gathered}
$$

where, $\rho^{2}=r^{2}+a^{2} \cos ^{2} \theta$; $\Delta=r^{2}-2 M r+a^{2}$

$M-$ is the mass of the black hole and $a$-is its rotation.

To calculate the force expression, one should know non-vanishing components of the Christoffel symbols $\Gamma_{k l}^{i}$. In this paper we only consider the case of the equatorial plane $=\pi / 2$. In this case nonvanishing Christoffel symbols are given by:

$$
\begin{aligned}
& \Gamma_{01}^{0}=\frac{M r^{2}\left(a^{2}+r^{2}\right)}{r^{2}\left(a^{2}+r^{2}\right)\left(r^{2}-2 M r\right)+2 M r a} \\
& \Gamma_{02}^{0}=\frac{-a^{2}\left(a^{4} M+2 r^{3}\left(M r-2 M^{2}\right)+a^{2}\left(3 M r-2 M^{2}\right)\right)}{2 r\left(r^{2}\left(a^{2}+r^{2}\right)\left(r^{2}-2 M r\right)+2 M r a\right)} \\
& \Gamma_{13}^{0}=\frac{-a M r^{2}\left(a^{2}+3 r^{2}\right)}{r^{2}\left(a^{2}+r^{2}\right)\left(r^{2}-2 \mathrm{Mr}\right)+2 M r a} \\
& \Gamma_{00}^{1}=\frac{M \Delta}{r^{4}} \\
& \Gamma_{03}^{1}=-\frac{a M \Delta}{r^{4}} \\
& \Gamma_{11}^{1}=\frac{a^{2}-M r}{r \Delta} \\
& \Gamma_{22}^{1}=-\frac{\Delta}{r} \\
& \Gamma_{33}^{1}=\frac{-\Delta\left(2 r-\frac{2 a^{2} M}{r^{2}}\right)}{r^{2}} \\
& \Gamma_{03}^{2}=\frac{a M\left(a^{2}+r^{2}\right)}{r^{5}} \\
& \Gamma_{12}^{2}=\frac{1}{r} \\
& \Gamma_{01}^{3}=\frac{a M}{r^{2}\left(a^{2}+r^{2}\right)\left(r^{2}-2 \mathrm{Mr}\right)+2 M r a} \\
& \Gamma_{31}^{3}=\frac{r\left(r^{3}(r-2 M)-a^{2} M r\right)}{r^{3}(r-2 M)\left(a^{2}+r^{2}\right)+2 a^{2} M r}
\end{aligned}
$$

From (2.2) by using formula:

$$
\frac{d^{2} x^{i}}{d \tau^{2}}+\Gamma_{k l}^{i} \frac{d x^{k}}{d \tau} \frac{d x^{l}}{d \tau}=0
$$


one can easily write the geodesic equation. We are interested only in the radial equation. In the Kerr metric it is given by:

$$
\begin{gathered}
\frac{d^{2} r}{d \tau^{2}}=-\frac{M \Delta}{r^{4}}\left(\frac{d t}{d \tau}\right)^{2}+\frac{2 a M \Delta}{r^{4}} \frac{d t}{d \tau} \frac{d \varphi}{d \tau}+\frac{M r-a^{2}}{r \Delta}\left(\frac{d r}{d \tau}\right)^{2} \\
+\frac{\Delta\left(2 r-\frac{2 a^{2} M}{r^{2}}\right)}{r^{2}}\left(\frac{d \varphi}{d \tau}\right)^{2},
\end{gathered}
$$

(where $\tau$ is the proper time)

In the left-hand side we have a radial acceleration. So, in the right-hand side one should have the radial component $F_{r}$ of the three force divided by the mass of the test body $m$.

However, one should pay attention only to components $\Gamma_{00}^{1}\left(\frac{d t}{d \tau}\right)^{2}=\frac{M \Delta}{r^{4}}\left(\frac{d t}{d \tau}\right)^{2}$ and $2 \Gamma_{03}^{1} \frac{d t}{d \tau} \frac{d \varphi}{d \tau}=-2 \frac{a M \Delta}{r^{4}} \frac{d t}{d \tau} \frac{d \varphi}{d \tau}$ (Landau, Lifshitz 1976).

The components $\Gamma_{11}^{1}\left(\frac{d r}{d \tau}\right)^{2}, \Gamma_{33}^{1}\left(\frac{d \varphi}{d \tau}\right)^{2}$ are not forces because they are the part of the three-covariant derivative (as we know from the differential geometry course, the derivative in general case does not obey the tensor law transformation).

In the limit of $a \rightarrow 0$ the leading term $\Gamma_{00}^{1}\left(\frac{d t}{d \tau}\right)^{2}$ in the geodesic equation (2.4) becomes well-known $\Gamma_{00}^{1}$ in the Schwarzschild spacetime $\Gamma_{00}^{1}=\frac{M}{r^{2}}\left(1-\frac{2 M}{r}\right)$ which corresponds to the Newton force of the
gravity attraction.

Comparing to the Schwarzschild case one can find that in the case of the equatorial plane $\theta=\pi / 2$ the leading term $\Gamma_{00 \text { Kerr }}^{1}$ in the Kerr metric has the following connection to the leading term in Schwarzschild spacetime $\Gamma_{00}^{1}$ sch

$$
\Gamma_{00 \mathrm{Kerr}}^{1}=\Gamma_{00 \mathrm{sch}}^{1}+\frac{a^{2} M}{r^{4}}
$$

From this equation one can easily see that in the limit $\mathrm{a} \rightarrow 0$ we have $\Gamma_{00 \mathrm{Kerr}}^{1}=\Gamma_{00 \mathrm{sch}}^{1}$ as we mentioned above. Also, one should notice that $\Gamma_{00 \mathrm{Kerr}}^{1} \geq \Gamma_{00 \text { sch }}^{1}$. The term $2 \Gamma_{03}^{1} \frac{d t}{d \tau} \frac{d \varphi}{d \tau}=2 \frac{-a M \Delta}{r^{4}} \frac{d t}{d \tau} \frac{d \varphi}{d \tau}$ corresponds to the Coriolis force. This type of force is absent in Schwarzschild spacetime because $\Gamma_{03}^{1}=0$ if $a \rightarrow 0$ pends upon the angular momentum of the black hole.

The main goal of this paper is to find out which forces act on the particle with zero energy. To find the particle energy one should write down the Lagrangian in Kerr spacetime which has the following form:

$$
L=\frac{1}{2} g_{i k} \frac{d x^{i}}{d \tau} \frac{d x^{k}}{d \tau}
$$

From this Lagrangian (2.6) one can easily obtain the energy $E$ and angular momentum $L$.

$$
\begin{aligned}
& E=-\frac{d L}{d \dot{t}}=\left(1-\frac{2 M}{\mathrm{r}}\right) \frac{d t}{d \tau}+\frac{2 M a}{\mathrm{r}} \frac{d \varphi}{d \tau} \\
& L=\left(r^{2}+a^{2}+\frac{2 M a^{2}}{r}\right) \frac{d \varphi}{d \tau}+\frac{2 M a}{\mathrm{r}} \frac{d t}{d \tau}
\end{aligned}
$$

From (2.8) one can easily see that the energy $E$ might be either negative or equal to zero in the ergosphere of a rotating black hole (e. g., in the region $M+\sqrt{M^{2}-a^{2}} \leq r \leq 2 M$ ). 


\section{Forces in the zero energy case}

In the previous section we found out that the energy in the ergosphere of a rotating black hole might be either negative or equal to zero. In this section we will consider the forces which act on the particle with zero energy. For this purpose, let us find out how the radial geodesic depends upon an energy $E$. Substituting (2.7), (2.8) into (2.4), one obtains:

$$
\begin{gathered}
\frac{d^{2} r}{d \tau^{2}}=-\frac{M \Delta}{r^{4}}\left(\frac{d t}{d \tau}\right)^{2}+\frac{2 a M \Delta}{r^{4}} \frac{d t}{d \tau} \frac{d \varphi}{d \tau}+\frac{M r-a^{2}}{r \Delta}\left(\frac{d r}{d \tau}\right)^{2} \\
+\frac{\Delta\left(2 r-\frac{2 a^{2} M}{r^{2}}\right)}{r^{2}}\left(\frac{d \varphi}{d \tau}\right)^{2}= \\
=-E \frac{M}{r^{2}} \frac{d t}{d \tau}-\frac{M a^{2}}{r^{4}}\left(\frac{d t}{d \tau}\right)^{2}+2 a M\left(\frac{r^{2}-M r+a^{2}}{r^{4}}\right) \frac{d t}{d \tau} \frac{d \varphi}{d \tau} \\
+\frac{M r-a^{2}}{r \Delta}\left(\frac{d r}{d \tau}\right)^{2}+\frac{\Delta\left(2 r-\frac{2 a^{2} M}{r^{2}}\right)}{r^{2}}\left(\frac{d \varphi}{d \tau}\right)^{2}
\end{gathered}
$$

Now, if we put $E=0$ in (3.1), we obtain the second order radial geodesic equation for the particle with zero energy:

$$
\begin{aligned}
\frac{d^{2} r}{d \tau^{2}}=-\frac{M a^{2}}{r^{4}}\left(\frac{d t}{d \tau}\right)^{2}+ & 2 a M\left(\frac{r^{2}-M r+a^{2}}{r^{4}}\right) \frac{d t}{d \tau} \frac{d \varphi}{d \tau}+\frac{M r-a^{2}}{r \Delta}\left(\frac{d r}{d \tau}\right)^{2} \\
& +\frac{\Delta\left(2 r-\frac{2 a^{2} M}{r^{2}}\right)}{r^{2}}\left(\frac{d \varphi}{d \tau}\right)^{2}
\end{aligned}
$$

As one can see, we have $\frac{M a^{2}}{r^{4}}\left(\frac{d t}{d \tau}\right)^{2}$ from the leading term $\Gamma_{00}^{1}\left(\frac{d t}{d \tau}\right)^{2}$. This is the same term in which the Kerr leading term differs from the Schwarzschild one. One can notice that in the case of small rotation $a^{2} M^{2}$ the leading term is negligible. Thus, the analogue the Newtonian force of attraction is negligible for the particles with zero energy and small rotation. However, as one can see from Fig. 1, the leading term $\Gamma_{00}^{1}$ if $E=0$ is bigger than the one with positive energy.

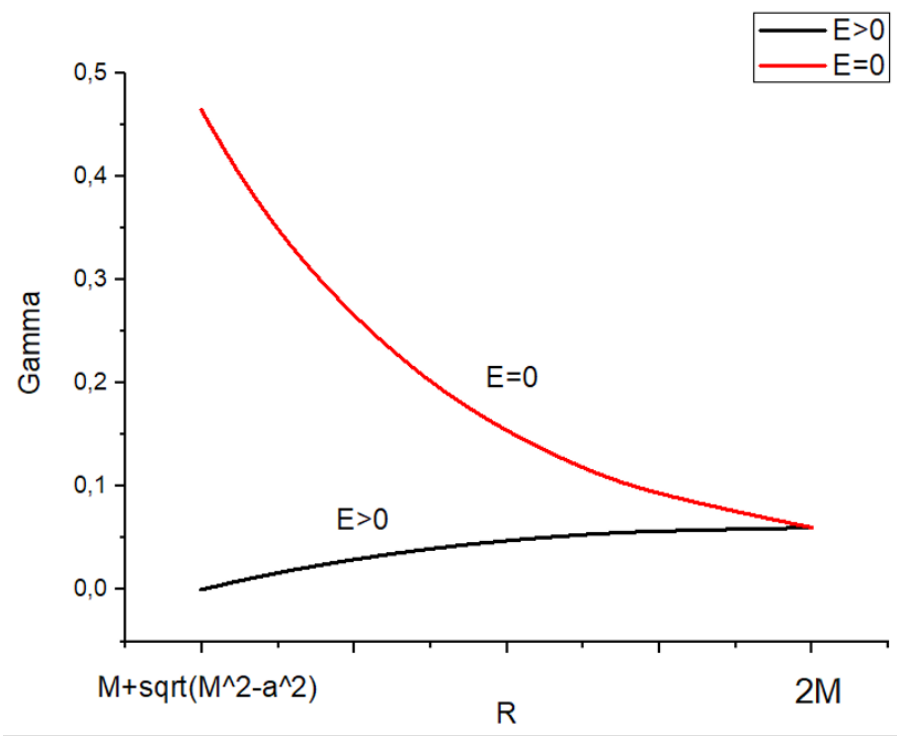

Fig. 1. Leading term 
However, if we compare the Coriolis force for usual particle with positive energy and one with zero energy, we can see that for zero energy case the Coriolis force is bigger than in the case of the positive force (Fig. 2). We can state this because we have the main condition-the movement forward in time i. g. $\frac{d t}{d \tau}$ must be positive. This condition demands the positivity of $\frac{d \varphi}{d \tau}$.

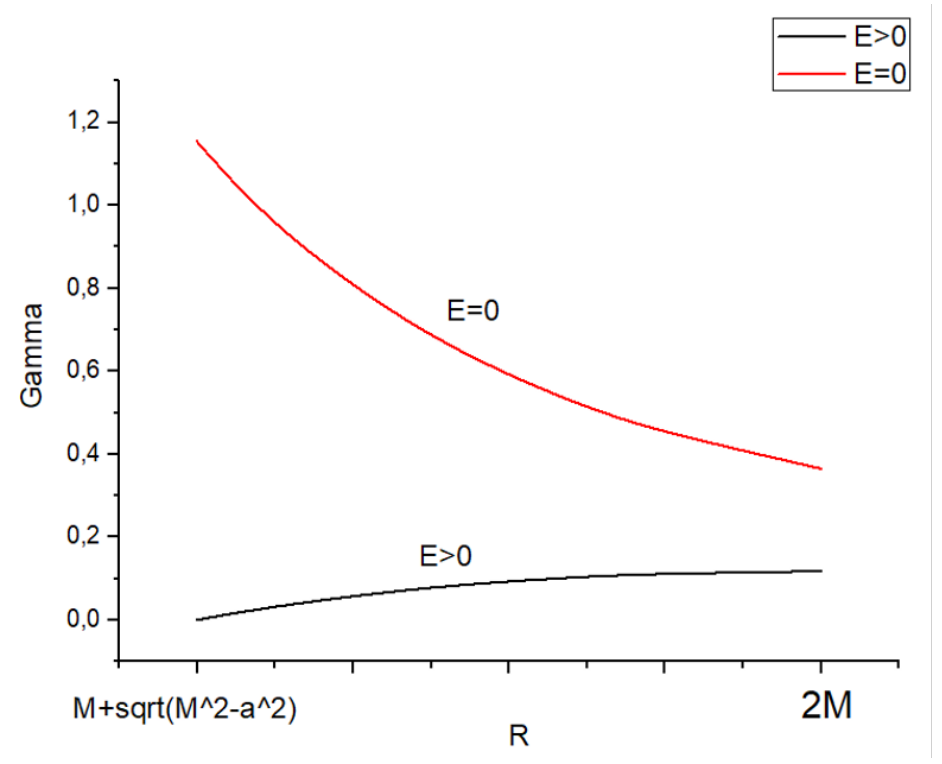

Fig. 2. Coriolis force

Conclusion

In this paper we have considered forces in the Kerr spacetime which act on the particles with zero energy. We showed that in the case of the leading term $\Gamma_{00}^{1}$ of the radial geodesic equation, in the case of zero energy and of small rotation, nearly vanishes and we have the term which shows how the leading term in the Kerr metric differs from the one in Schwarzschild spacetime. It is worth noticing that this force component for particle with zero energy is bigger than the one with positive energy. Regarding the Coriolis force, the radial component of this force in the case of zero energy is bigger than the same component in the case of positive energy. The main result of this paper is that all force components for the particles with zero energy are bigger than the ones for particles with positive energy.

\section{Conflict of Interest}

The authors declare that there is no conflict of interest, either existing or potential.

\section{Reference}

Chandrasekhar, S. (1983) The mathematical theory of black holes. New York: Clarendon Press, 646 p. (In English) Grib, A. A., Pavlov, Yu. V. (2017) Black holes and particles with zero or negative energy. Theoretical and Mathematical Physics, 190 (2), 268-278. https://doi.org/10.1134/S0040577917020088 (In English)

Grib, A. A., Pavlov, Yu. V., Vertogradov, V. D. (2014) Geodesics with negative energy in the ergosphere of rotating black holes. Modern Physics Letters A, 29 (20), 14501-14510. https://doi.org/10.1142/S0217732314501107 (In English)

Landau, L. D., Lifshitz, E. M. (1976) Course of Theoretical Physics Series: In 10 vols. Vol. 2. The classical theory of fields. $4^{\text {th }}$ ed. Oxford: Butterworth-Heinemann Publ., 444 p. (In English)

Penrose, R. (2002) "Golden Oldie": Gravitational collapse: The role of general relativity. General Relativity and Gravitation, 34 (7), 1141-1165. https://doi.org/10.1023/A:1016578408204 (In English)

Poisson, E. (2004) A relativist's toolkit: The mathematics of black-hole mechanics. Cambridge: Cambridge University Press, 233 p. https://doi.org/10.1017/CBO9780511606601 (In English)

Teukolsky, S. A. (2015) The Kerr metric. Classical and Quantum Gravity, 32 (12), article 124006 https://doi.org/10.1088/0264-9381/32/12/124006 (In English)

Vertogradov, V. D. (2015) Geodesics for particles with negative energy in Kerr's metric. Gravitation and Cosmology, 21 (2), 171-174. https://www.doi.org/10.1134/S0202289315020115 (In English)

Vladimirov, Yu. S. (2009) Klassicheskaya teoriya gravitatsii [Classical gravity theory]. Moscow: Librokom Publ., 264 p. (In Russian) 